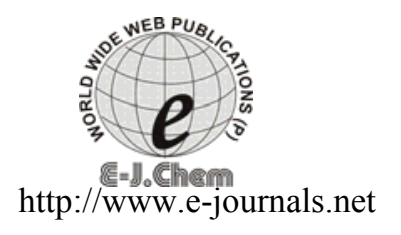

ISSN: 0973-4945; CODEN ECJHAO

E-Journal of Chemistry

2011, 8(S1), S105-S112

\title{
Mechanical, Thermal and Surface Investigations of Chitosan/Agar/PVA Ternary Blended Films
}

\author{
ESAM A. EL-HEFIAN ${ }^{*}$, MOHAMED MAHMOUD NASEF and \\ ABDUL HAMID YAHAYA ${ }^{\S}$ \\ ${ }^{*}$ Chemistry Department, Faculty of Science, \\ University of the Western Mountain, Azzawiyah, Libya \\ Chemical Engineering Department \\ Faculty of Chemical and Natural Resources Engineering \\ Universiti Teknologi Malaysia, 81310 UTM Skudai, Johor, Malaysia \\ ${ }^{\S}$ Department of Chemistry, Faculty of Science \\ University of Malaya, 50603 Kuala Lumpur, Malaysia \\ eelhefian@yahoo.com
}

Received 26 February 2011; Accepted 27 April 2011

\begin{abstract}
The mechanical and thermal properties of chitosan/agar/poly vinyl alcohol (CS/AG/PVA) ternary blended films having various proportions considering chitosan as the main component were investigated. The various variables static water contact angle such as contact angle, drop base area, drop volume and drop height was also studied in correlation with the variation of time. Results obtained from mechanical measurements showed a noticeable increase in the tensile strength (TS) coincided with a sharp decrease in elongation percent at break (E\%) of blended films with increasing agar and PVA contents. The DSC results prevailed the development of an interaction between chitosan individual components: agar and PVA. Moreover, an enhancement of the wettability of the blends was obtained with increasing agar and PVA contents. It was also found that the pure CS film and the blended films with 90/05/05 and 80/10/10 compositions were more affected by time than blended films with other compositions when the contact angle, the drop height and the drop length were studied as a function of time. In addition, when the drop is initially placed on the substrate, the drop area and the drop volume of all films remained almost constant up to a certain time after which they showed a slight difference with the elapse of time.
\end{abstract}

Keywords: Chitosan, Agar, PVA, Blend, Film

\section{Introduction}

Natural polymers have attracted an increasing attention over the last two decades, mainly due to their abundance, environmental concerns and anticipated depletion of petroleum resources. 
This has led to a growing efforts in developing chemical and biochemical processes to obtain and modify natural polymers and utilize their inherent properties for a wide range of applications in different fields ${ }^{1,2}$. Blending of natural polymers with synthetic ones is an interesting method to obtain new materials in which the former properties are improved to suit particular applications ${ }^{3}$. Among natural polymers, chitosan occupies a special position duo to its versatility in that it enhances its medicinal value by blending with synthetic and natural polymers to its extended applications. Recently, some ternary blended films, such as chitosan/ PVA/gelatin ${ }^{4}$ and chitosan/PVA/pectin ${ }^{5}$ were reported. However and up to the knowledge of the authors, reports on modification of chitosan by blending with agar and PVA in a ternary blend have been very scarce. A combination of these three polymers is expected to lead to chitosan based films with improved properties including degree of swelling, tensile strength and surface wettability as a result of their interactions by hydrogen bonding.

In previous articles, we studied the effects of agar and PVA contents separately on the properties of chitosan at different ratios considering chitosan as the major component $^{6-9}$. In this study, we report on the preparation of chitosan/agar/PVA ternary blended films obtained by physical solution blending of chitosan, agar and PVA at various proportions with chitosan representing major components using the same amount of agar and PVA. The mechanical, thermal (measured by DSC) and surface (determined by contact angle measurements) properties of these ternary blended films were also investigated.

\section{Experimental}

Shrimp source chitosan with a degree of deacetylation (DD) of $88.1 \%$ determined by UV method ${ }^{10}$ was purchased from a commercial source. The agar was purchased from Sigma and its average molecular weight was $1.3 \times 10^{4} \mathrm{~g} \mathrm{~mol}^{-1}$. The PVA from Fluka (56-98) had an average molecular weight of $195 \times 10^{3} \mathrm{~g} \mathrm{~mol}^{-1}$. Acetic acid (glacial 100\%, pro-analysi) was purchased from Merck (Darmstadt, Germany). Ultra pure water (Maxima Ultra Pure Water, Elga-Prima Corp, UK) with a resistivity greater than $18 \mathrm{M} \Omega / \mathrm{cm}$ was used to prepare all solutions. All chemicals were used without any further purification.

\section{Preparation of CS/AG/PVA ternary blended solutions}

$10 \mathrm{~g} \mathrm{~L}^{-1}$ solution of CS was prepared by dissolving $5 \mathrm{~g}$ of chitosan in $500 \mathrm{~mL}$ acetic acid $(0.1 \mathrm{M})$ (using a volumetric flask) followed by stirring and heating at $60{ }^{\circ} \mathrm{C}$ overnight. The solution was filtered to remove dust and other traces of impurities. To ensure a complete dissolution of $\mathrm{CS}$, the residue of filtration was attempted to be dissolved in acetic acid of the same concentration but it remained. Air bubbles were eliminated by keeping the solutions at room temperature for $2 \mathrm{~h}$. A similar $10 \mathrm{~g} \mathrm{~L}^{-1}$ solution of $\mathrm{AG}$ as well as PVA were prepared by dissolving $5 \mathrm{~g}$ of each polymer in $500 \mathrm{~mL}$ preheated ultrapure water. The solutions was then stirred and kept at about $90{ }^{\circ} \mathrm{C}$ for $2 \mathrm{~h}$. Then, CS and AG solutions were blended together under continuous stirring at around $94{ }^{\circ} \mathrm{C}$ for $30 \mathrm{~min}$ to form a homogenous blend solution to ensure that the added components particularly AG remain in a liquid form during blending. Finally, CS, AG and PVA solutions were homogenously blended at $94{ }^{\circ} \mathrm{C}$ for $30 \mathrm{~min}$ to form ternary blended solutions having various selected compositions as presented in Table 1 taking into consideration CS as the major component in the obtained blends. 
Table 1. The different compositions of the chitosan, agar and PVA ternary blends

\begin{tabular}{cccc}
\hline $\begin{array}{c}\text { Blend compositions } \\
\text { (CS/AG/PVA) }\end{array}$ & wt \%, CS & wt \%, ${ }_{\text {AG }}$ & wt \%, PVA \\
\hline $90 / 05 / 05$ & 90 & 5 & 5 \\
$80 / 10 / 10$ & 80 & 10 & 10 \\
$70 / 15 / 15$ & 70 & 15 & 15 \\
$60 / 20 / 20$ & 60 & 20 & 20 \\
$50 / 25 / 25$ & 50 & 25 & 25 \\
\hline
\end{tabular}

\section{Preparation of CS/AG/PVA ternary blended films}

The ternary blended solutions $(10 \mathrm{~mL}$ each) were poured onto polystyrene petri dishes followed by drying at $60{ }^{\circ} \mathrm{C}$ for $48 \mathrm{~h}$. The resultant blended films were peeled off gently and kept under evacuated desiccator over fresh silica gel until use. All films obtained were transparent and free of air bubbles.

\section{Film thickness}

The film thickness was measured with a digital micrometer (Mitutoyo, Japan) with $0.001 \mathrm{~mm}$ resolution. Several thickness measurements were taken at several points of the film and then, the average was calculated. The thicknesses of the films were found to be around $15 \mu \mathrm{m}$.

\section{Methods}

\section{Molecular weight measurements}

The molecular weight of chitosan was $5.5 \times 10^{5} \mathrm{~g} \cdot \mathrm{mol}^{-1}$ as determined by gel permeation chromatography (GPC) using the procedure described elsewhere ${ }^{11}$.

\section{Mechanical properties}

The mechanical properties were measured using a universal mechanical tester (Instron, Model 5566, USA) according to the ASTM D 882-91. Dumbbell-shaped specimens of $50 \mathrm{~mm}$ length with a neck of 28 and $4 \mathrm{~mm}$ width were used. The measurements were carried out at $23{ }^{\circ} \mathrm{C}$ and $50 \%$ relative humidity and at crosshead speed of $50 \mathrm{~mm} / \mathrm{min}$. A minimum of five specimens were tested for each sample and the average was recorded.

\section{DSC measurements}

Differential scanning calorimetric (DSC) studies were performed using a DSC Mettler Toledo (model DSC822e). The samples were scanned under a nitrogen atmosphere in a temperature range of $40-150{ }^{\circ} \mathrm{C}$ at a constant heating rate of $10{ }^{\circ} \mathrm{C} / \mathrm{min}$ in two-heating runs.

\section{Contact angle measurements}

The static water contact angles of the films were measured at room temperature by the drop method using an optical contact angle meter CAM 200 (KSV Instruments Ltd, Helsinki, Finland) to examine the surface wettability of the films. The substrates used for the experiments were glass microscope slides $(25.4 \times 76.2 \mathrm{~mm}, 1-1.2 \mathrm{~mm}$ thick). Each slide was cleaned before use by soaking in ethanol overnight. $7 \mu \mathrm{L}$ of distilled water was carefully injected on the film surface before conducting the measurement which extended to 24 second. The contact angles were measured on both sides of the drop and averaged. Each reported contact angle represents the mean value of at least 10 measurements. 


\section{Results and discussion}

\section{Tensile strength and \% of elongation at break}

Figures 1 and 2 present the tensile strength and the \% of elongation at break of the $\mathrm{CS} / \mathrm{AG} / \mathrm{PVA}$ blended films with different AG and PVA proportions respectively. As it can be seen, all the CS/AG/PVA ternary blended films recorded higher values of tensile strength than the pure chitosan. The TS increased gradually as the amounts of AG and PVA increased until the ratio 60/20/20, which recorded the highest value of TS (50.76 MPa) then the TS decreased slightly. This improvement in TS could possibly be due to the interaction between $-\mathrm{OH}$ and $-\mathrm{NH}_{2}$ groups originated from chitosan and $-\mathrm{OH}$ groups present in agar and PVA. On contrary, E\% was found to sharply drop upon blending with AG and PVA regardless of their concentrations compared to that of pure CS recorded suggesting an increase brittleness of the blended films despite the increase in their strength.

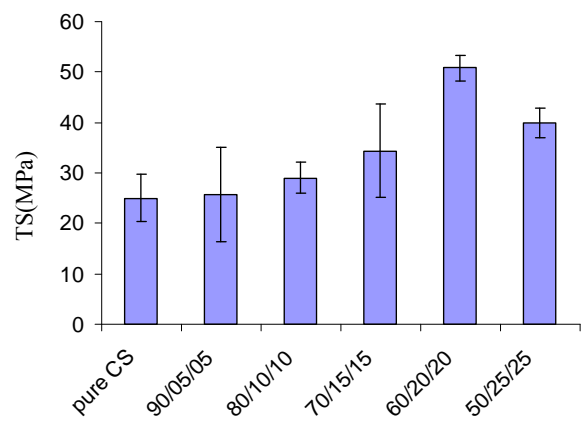

Figure 1. Tensile strength of the pure CS and its ternary blended films with AG and PVA at various proportions

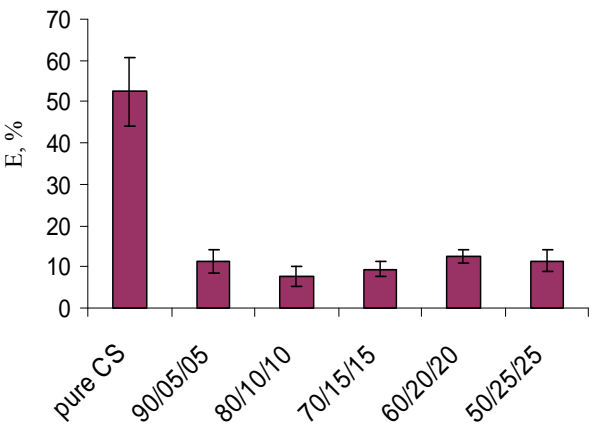

Figure 2. Elongation \% of the pure CS and its ternary blended films with AG and PVA at various proportions

\section{DSC measurements}

Figure 3 depicts the DSC curves of the blended CS/AG/PVA films together with their pure CS film. As can be seen, the DSC first run curve of CS film shows a broad endothermic peak at about $79{ }^{\circ} \mathrm{C}$, which shows agreement with previous studies ${ }^{12,13}$. This peak is attributed to the bound water in the film. The ternary blended films exhibited broad endothermic peaks in the range of 77.3 to $79.6{ }^{\circ} \mathrm{C}$ (Table 2). This was combined with the absence of any significant difference in the endothermic peak areas of the films, i.e., blended films do not vary much in their water-holding capacity. Another point to be noted is that all ratios have lower values of $T_{\mathrm{D}}$ (the dehydration temperature) than the pure CS except the ratio $70 / 15 / 15$, which showed higher $T_{\mathrm{D}}$.

This variation on the position could be due to the physical and molecular changes caused by the molecular chains interaction between the CS, AG and PVA, which leads to form more stable films

Figure 4 shows the second heating run of the DSC, which was conducted to decrease the water content in the films. The first point to note is the absence of the endothermic peak confirming that this peak is attributed to the water content in the samples. Also, smaller peaks of melting transition at different temperatures appeared in the DSC curves of $\mathrm{CS} / \mathrm{AG} / \mathrm{PVA}$ blended films with various blend ratios ranging from 217.3 to $219.6{ }^{\circ} \mathrm{C}$ as shown in Table 2. 


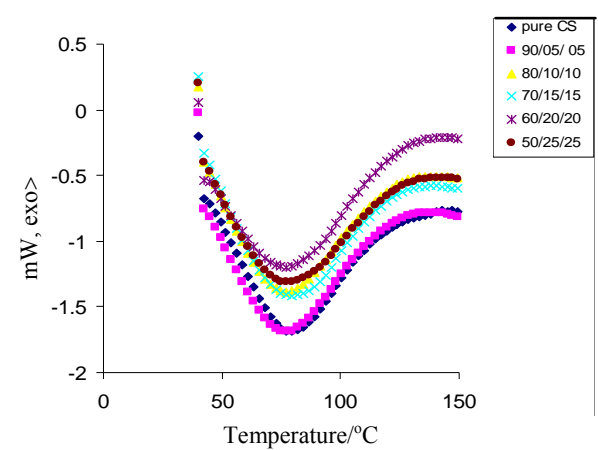

Figure 3. The DSC first run curves of the pure CS and its ternary blended films with AG and PVA having various proportions

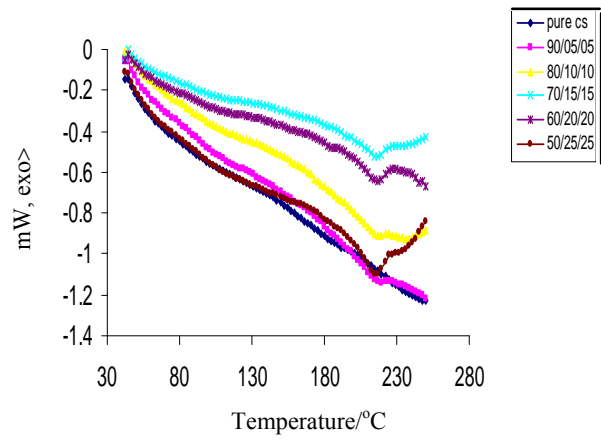

Figure 4. The DSC second run curves of the pure CS and its ternary blended films with AG and PVA having various proportions

Table 2. The thermal properties of the pure CS and its ternary blended films with AG and PVA having various proportions

\begin{tabular}{ccccccc}
\hline $\begin{array}{c}\text { Blend } \\
\text { composition }\end{array}$ & $100 / 0 / 0$ & $90 / 05 / 05$ & $80 / 20 / 20$ & $70 / 15 / 15$ & $60 / 20 / 20$ & $50 / 25 / 25$ \\
CS/AG/PVA & & & & & & \\
\hline $\mathrm{T}_{\mathrm{D}}$ (first run) & 79.0 & 77.3 & 77.3 & 79.6 & 77.3 & 77.3 \\
$\Delta \mathrm{H}(\mathrm{J} / \mathrm{g}$ ) (first run) & 227.6 & 244.1 & 255.7 & 283.9 & 214.3 & 246.1 \\
$\mathrm{~T}_{\mathrm{m}}$ (second run) & $\mathrm{ND}$ & 219.6 & 219.6 & 217.3 & 217.3 & 219.6 \\
\hline
\end{tabular}

\section{Contact angle measurements (surface hydrophilicity)}

Figure 5 shows the static water contact angle of the pure CS film and its ternary blended films with AG and PVA at various proportions. The static contact angle of the pure CS was found to be $88^{\circ}( \pm 1.91)$, which is in a good agreement with the literature ${ }^{14,15}$. Whereas, the contact angle of the blended films were found to be in the range of $88^{\circ}( \pm 2.15)$ to $80^{\circ}( \pm 0.75)$ (less than $90^{\circ}$ ) indicating the remaining of a great deal of hydrophobicity levels of the surfaces of the obtained blended films. It was also found that there is almost no change in the static water contact angle up to $10 \%$ of AG or PVA beyond which a decreasing trend is obtained with the increase in AG or PVA content in the blend i.e., an increase in the wettability of the surface of the ternary blended films can be achieved upon having AG and PVA contents in the range of $15-25 \%$. The nearly constant values of static water contact angle are most likely due to the dominance of chitosan at film surface despite the presence of $10 \%$ AG or PVA. However, as minor components concentration increase, more hydrophilicity is imparted to the blended films surfaces causing an improvement in the wettability of the films i.e. reduction in static water contact angle.

Figure 6a shows the variation in the water contact angle with time for the pure CS and its ternary blended films with AG and PVA having different proportions. The contact angle of the pure CS film and that of 90/05/05 and 80/10/10 ternary films decreases rapidly after making the initial contact on the substrate. However, the ternary films of other concentrations showed a gradual decrease in contact angle with time. These results also showed that the addition of the AG and PVA to chitosan can increase the hydrophilic nature of chitosan. The change in contact angle with time could be due to evaporation, surface oxidation 
or dissolution ${ }^{16}$. Similar behaviour was observed when the drop height was studied as a function of time (Figure 6b). In addition, opposite behaviour was pronounced regarding the drop length versus time (Figure 6c).

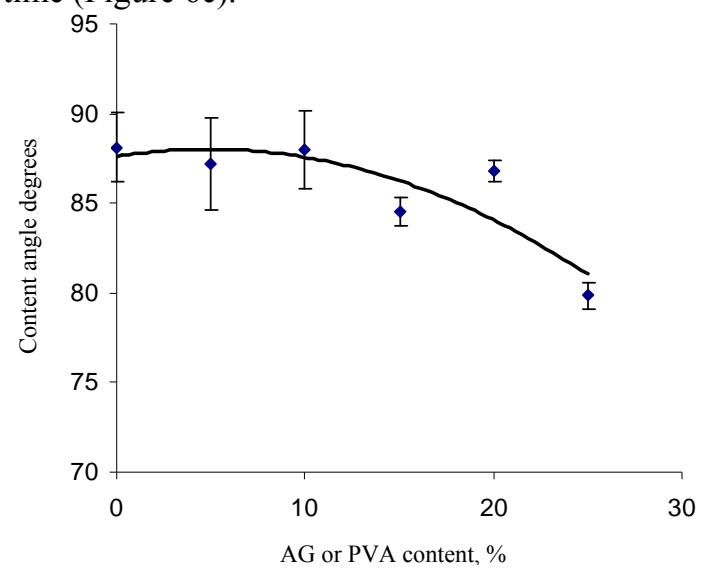

Figure 5. The relationship between the water-contact angle and the AG or PVA content $(0 \%=$ pure $\mathrm{CS})$

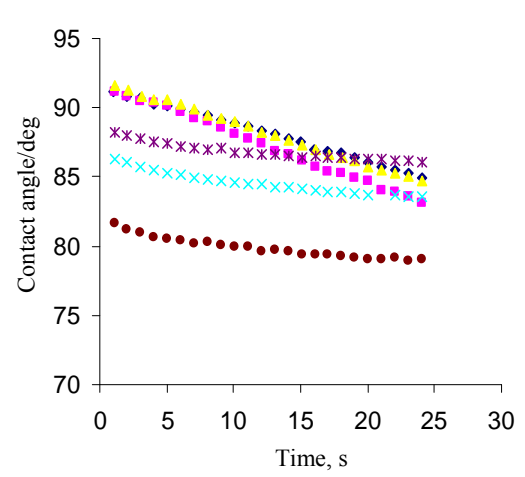

(a)

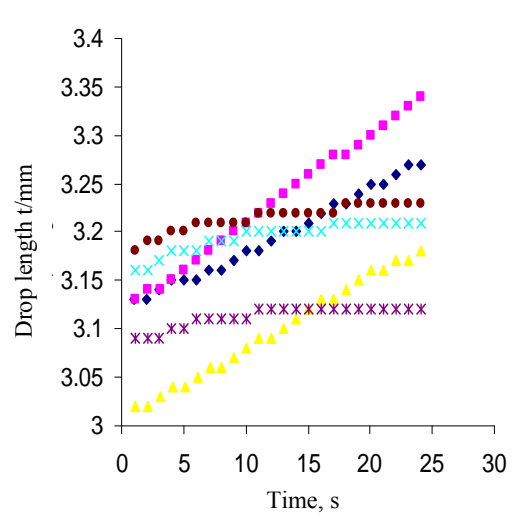

(c)
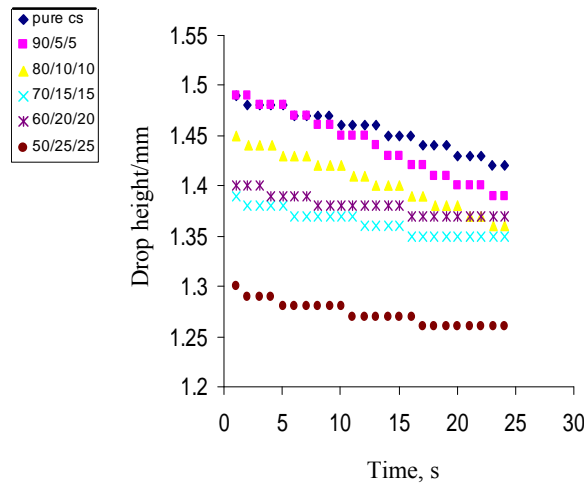

(b)
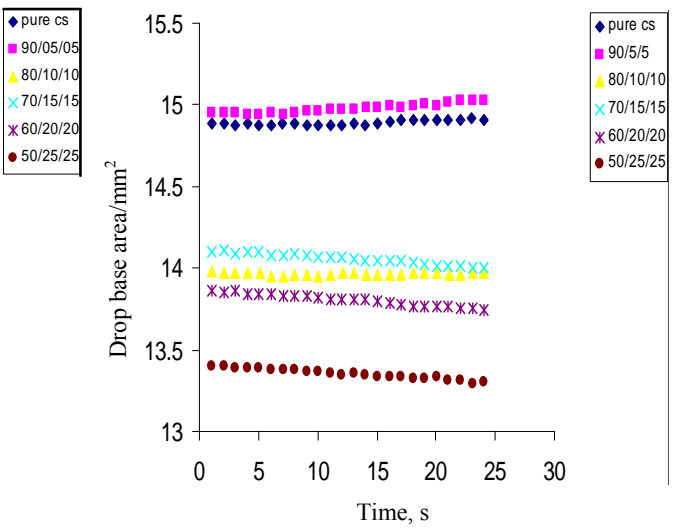

(d) 


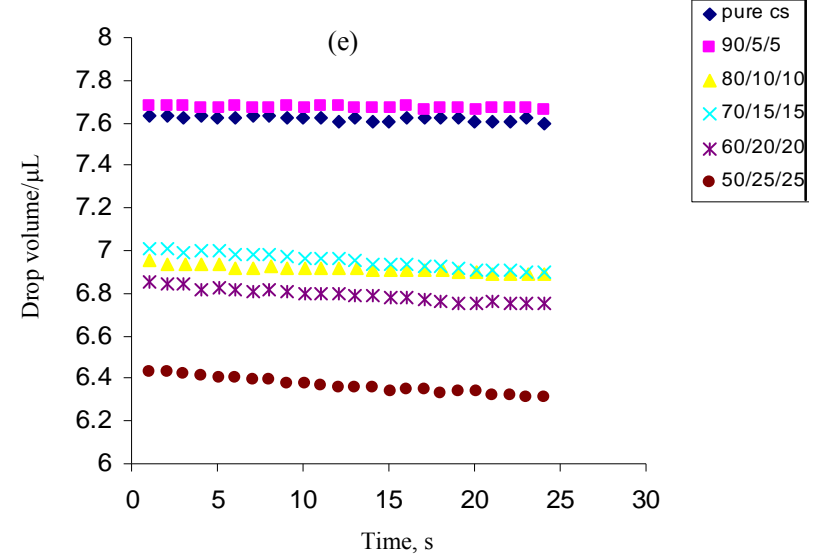

Figure 6. The variations of (a) contact angle, (b) drop height, (c) drop length, (d) drop base area and (e) drop volume with time

The drop base area is one of the important geometrical dimensions, which can be obtained along with other parameters. The results in terms of drop base area for the ternary blends are shown in Figure 6d. When the drop is initially placed on the substrate, the drop area remains constant up to certain time after which it showed a slight increase with respect to the pure CS film together with $90 / 05 / 05$ and $80 / 10 / 10$ ternary films whereas a slight decrease in the rest of the ternary films could be observed.

However, the drop volume remains almost constant for the pure CS together with $90 / 05 / 05$ and $80 / 10 / 10$ ternary films while showing a very slow decrease with the elapse of time for the rest of the films (Figure 6e). This suggests that the volume change is mostly due to evaporation. Nevertheless, such evaporation takes place depending on the level of hydrophilicity which increases with the increase of AG or PVA in blend from $15-25 \%$.

\section{Conclusion}

Ternary blended films of chitosan/agar/poly vinyl alcohol (CS/AG/PVA) were prepared at various proportions. The properties of the obtained blended films were determined using universal mechanical tester, DSC and contact angle measurements. Results revealed that the mechanical properties of the blended films showed an increase in the tensile strength coupled with a decrease in the elongation \% at break with the increase in the content of the agar and PVA. The DSC analysis showed that the interaction between chitosan, agar and PVA mostly taken place. It was also found that better wettability is obtained with increasing agar and PVA contents. In addition, the pure CS film and the blended films with 90/05/05 and 80/10/10 ratios were found to be more affected by time than the other blended films when the contact angle, the drop height and the drop length are studied as a function of time. However, when the drop is initially placed on the substrate, the drop area and the drop volume of all films showed a slight difference with the elapse of time.

\section{Acknowledgment}

The authors are grateful to University of Malaya for supporting this research project under the research grant, PS188/2008A. 


\section{References}

1. Illum L, Pharm Res., 1998, 15, 1326-1331.

2. Akbuga J, Int J Pharm Adv., 1995, 1, 1-18.

3. Yang X, Zhu Z, Liu Q, Chen X and Ma M, Radiat Phys Chem., 2008, 77, 954-960.

4. Chen C H, Wang F Y, Mao C F, W. Liao W T and Hsieh C D, Int J Biol Macromol., 2008, 43(1), 37-42.

5. Tripathi S, Mehrotra G K and Dutta P K, Carbohydr Polym., 2010, 79(3), 711-716.

6. El-hefian E A, Nasef M M, Yahaya A H and Khan R A, J Chil Chem Soc., 2010, 55, 130-136.

7. El-hefian E A, Nasef M M and Yahaya A, E-J Chem., 2010, 7(4), 1212-1219.

8. El-hefian E A, Nasef M M and Yahaya A H, E- J Chem., 2010, 7(S1), S349-S357.

9. El-hefian E A, Nasef M M and Yahaya A H, E- J Chem., 2010, 2011, 8(1), 91-96.

10. Muzzarelli R A A and Rochetti R, Carbohydr Polym., 1985, 5, 461-472.

11. El-hefian E A, Elgannoudi E S, Mainal A and Yahaya A H, Turk J Chem., 2010, 34(1), 47-56.

12. Lima C G A, De Oliveira R S, Figueiro S D, Wehmann C F, Goes Sombra J C and Ombra A S B, Mater Chem Phys., 2006, 99, 284-288.

13. Wang Y, Lin M, Wang D and Hsieh H, Biomaterials., 2003, 24(6), 1047-1057.

14. Tangpasuthadol V, Pongchaisirikul N and Hoven V P, Carbohydr Res., 2003, 338(9), 937-942.

15. De Britto D and Assis O B G, Carbohydr Polym., 2007, 69(2), 305- 310.

16. Raichur A. M, Wang X H and Parekh B K, Colloids Surf A Physicochem Eng Asp., 2000, 167(3), 245-251. 


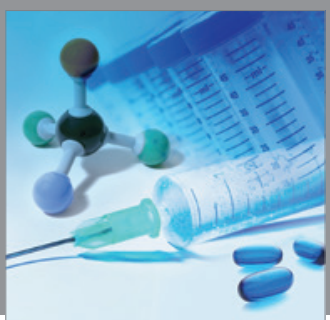

International Journal of

Medicinal Chemistry

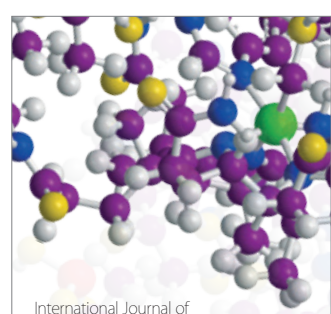

Carbohydrate Chemistry

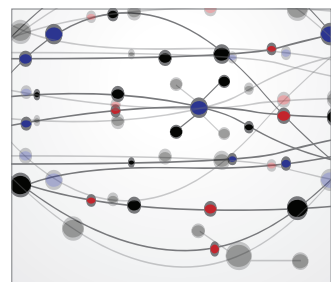

The Scientific World Journal
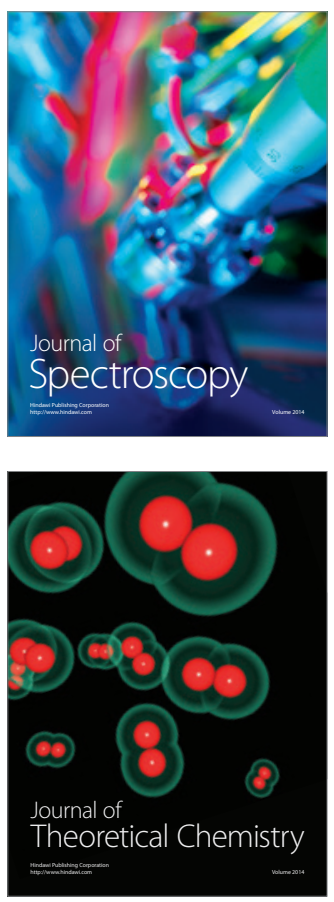
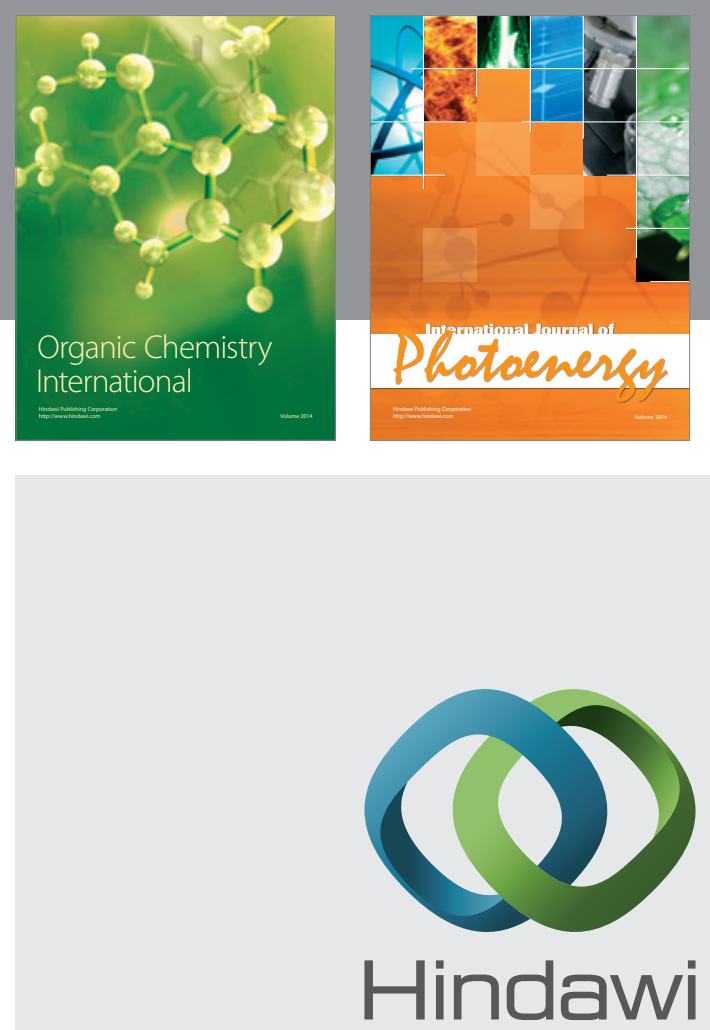

Submit your manuscripts at

http://www.hindawi.com
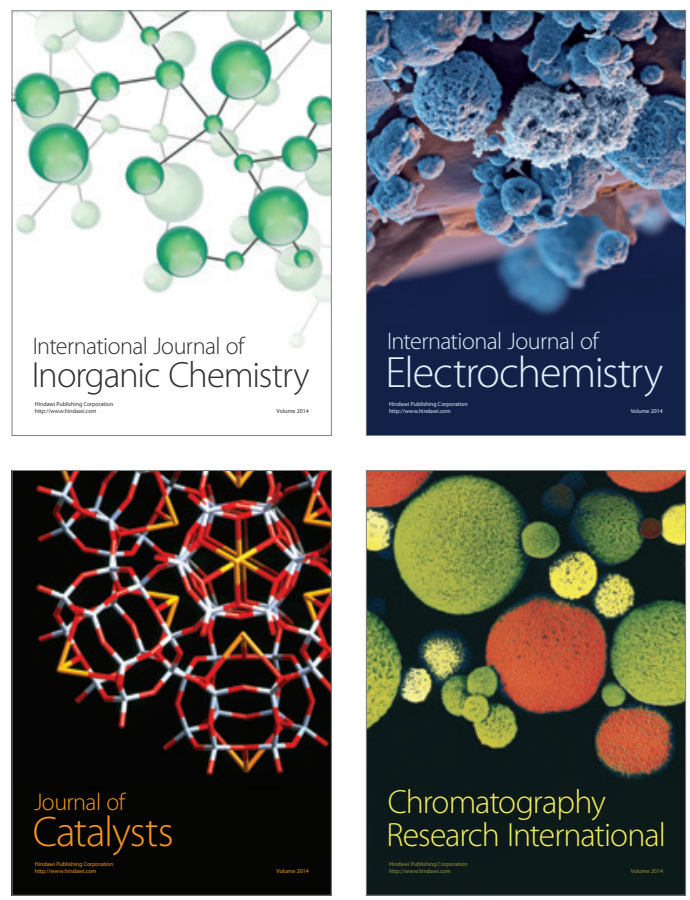
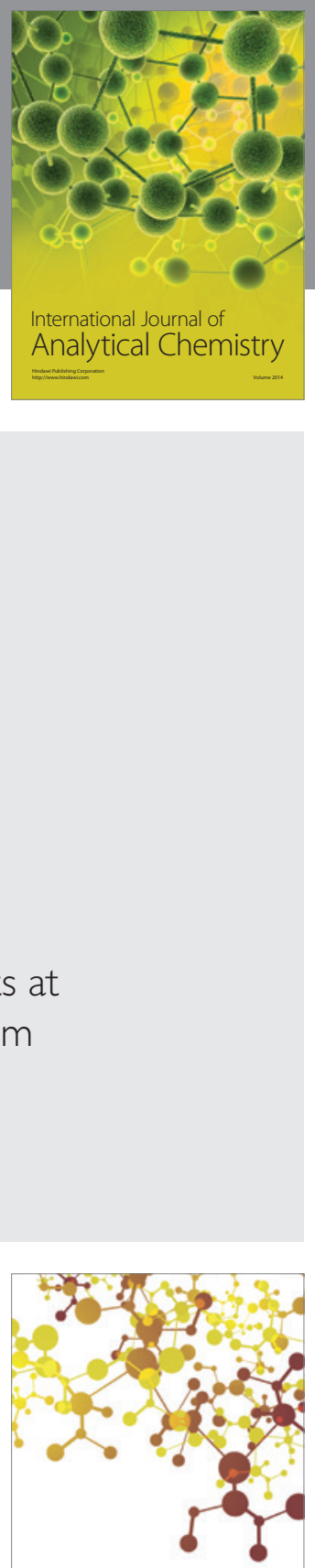

Journal of

Applied Chemistry
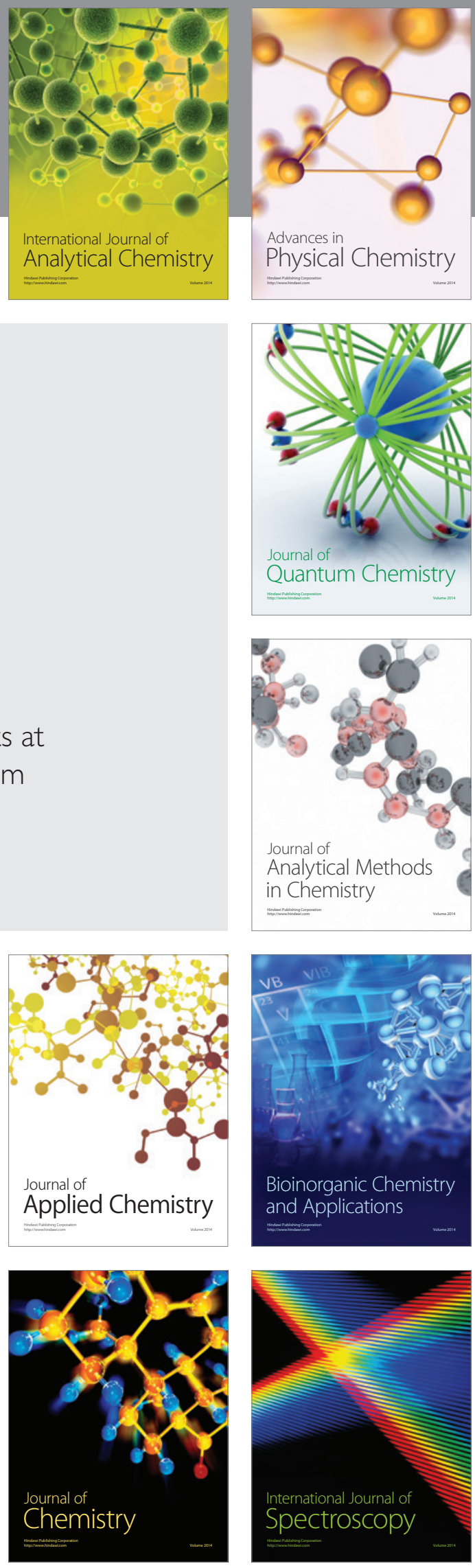